\title{
EFFECT OF SWIRLING GAS FLOW IN ELECTRODE CAVITY ON ITS EROSION
}

\author{
A.S. Anshakov \\ SB RAS \\ Kutateladze Institute of Thermophysics \\ Novosibirsk, Russia \\ anshakov@itp.nsc.ru \\ E.B. Butakov \\ SB RAS \\ Kutateladze Institute of Thermophysics \\ Novosibirsk, Russia \\ e_butakov@mail.ru \\ A.E. Urbakh \\ SB RAS \\ Kutateladze Institute of Thermophysics \\ Novosibirsk, Russia \\ urbakh@itp.nsc.ru
}

\author{
P.V. Domarov \\ Novosibirsk State Technical University \\ Novosibirsk, Russia \\ Domaroff@yandex.ru
}

\author{
V.A. Faleev \\ SB RAS \\ Kutateladze Institute of Thermophysics \\ Novosibirsk, Russia \\ v.faleev2010@yandex.ru \\ V.S. Cherednichenko \\ Novosibirsk State Technical University \\ Novosibirsk, Russia
}

\author{
V.R. Bower \\ Novosibirsk State Technical University \\ Novosibirsk, Russia
}

\begin{abstract}
Investigation results on specific erosion of copper tubular electrodes of the arc plasmatron for air heating are presented. The decisive influence of spatial stabilization of the arc discharge and current strength on the value of specific erosion is shown. The conditions of optimal erosion of electrodes are determined depending on the flow rate of plasma-forming gas, pressure in a discharge chamber, and an electrode diameter
\end{abstract}

Keywords- plasmatron, electrode, plasma-forming gas, electrode's erosion, arc current

The use of cupper cylindrical elecnrodes in the arc plasmatorches proved their high reliability and prospects for the heating of oxygen-bearing media. A relatively low level of specific erosion of these elecrodes $\left(2 \cdot 10^{-9} \mathrm{~kg} / \mathrm{C}\right.$ for cathodes and $2 \cdot 10^{-9} \div 5 \cdot 10^{-11} \mathrm{~kg} / \mathrm{C}$ for anodes) $[1,2]$ is provided by spreading of the heat flux from a bearing spot of the arc over a larger area because of its fast moving over the surface due to aerodynamic and electrodynamic forces, which act on a radial region of the arc. However, there are some regimes of arc combustion, when erosion of electrodes may be high and their lifetime is insignificant for practical use of the plasma device.
In plasmatorches with gas-vortex stabilization of the arc, considerable erasion of the inner cylindrical electrode is usually caused by incorrect choice of operation parameters (for instance, an insufficient flow rate of the working gas or excessively high current of the arc), which negatively affect the rate and dynamics of the arc spot movement.

The presented paper deals with criterion relationships between interconnected plasmatorch parameters, which provide minimal (optimal) erosion values for electrode material.

\section{EXPERIMENTAL SETUP}

The object of experimental study is internal hollow electrode $l$ with diameter $d$ of a three-chamber electric-arc plasmatron with gas-vortex arc stabilization (Fig. 1). In this constructive scheme, diaphragm 3 separating the electrodes ensures independence of gas-dynamic parameters in the internal electrode from the events occurring in the output step electrode. The diameter of the inner cavity was $(20,50$ and $60) \cdot 10^{-3} \mathrm{~m}$. Polarity of connection to the power supply was direct (electrode-cathode) or reverse (electrode-anode). 
Observation of the dynamics of the arc spot motion was observed using a high-speed SKS-1M camera through quartz glass 4 in the back cover of plasmatron.

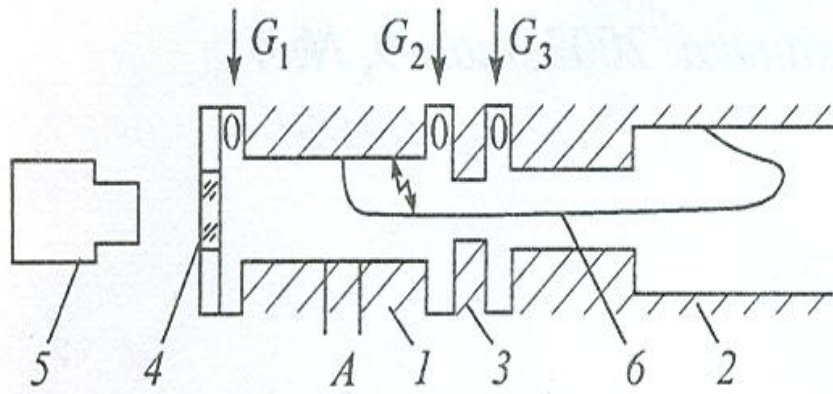

Fig. 1. Scheme of plasmatron with cylindrical internal electrode.

1, 2 - electrodes, 3 - interelectrode insert, 4 - quartz glass, 5 - camera, 6 $-\operatorname{arc}$

On the ends of the electrode, there are the swirling rings, through which air is supplied with flow rates $G_{1}$ and $G_{2}$ to control the near-electrode section and reference arc spot. An arc spot is located at the meeting point of flows $G_{1}$ and $G_{2}$ (ring zone $A$ ). The entrainment of eroded mass $\Delta m, \mathrm{~kg}$ is determined by weighing before and after the test with duration from 30 minutes to two hours, depending on the arc current strength. The value of specific erosion was calculated by ratio $\bar{G}=\Delta m /(I t)$, where $I$ is current strength, A; $t$ is test time, s.

\section{EROSION OF THE INNER CYLINDRICAL ELECTRODE}

Aerodynamics of the flow in the electrode cavity was investigated by a two-channel pneumometric sensor using the well-known technique. In cold and hot tests, distributions of static pressure $p$, circumferential $V_{\varphi}$ and axial $V_{z}$ velocity component of the flow of plasma-forming gas were measured. The flow rates of cooling water and plasma-forming air, arc current and voltage, and electrode weights were measured with the accuracy not worse than $\pm 1 \%$.

A priori, it is known that in plasmatrons with gas-vortex stabilization of an arc discharge in the absence of external magnetic field, the parameters of plasma-forming gas have a significant effect on dynamics of the near-electrode region, velocity of arc spot motion and electrode erosion.

Investigations of cathode specific erosion vs. arc discharge current have shown that in dependence $\bar{G}=f(I)$ at the level of $(1.5-2) \cdot 10^{-9} \mathrm{~kg} / \mathrm{K}$, there is a phenomenon of critical current $I_{c r}$, above which a sharp increase in $\bar{G}\left(5 \cdot 10^{-9} \mathrm{~kg} / \mathrm{C}\right.$ and higher) is observed [3,4]. If for electrode diameter $d=20 \cdot 10^{-}$ ${ }^{3} \mathrm{~m}, I_{c r} \approx 300 \mathrm{~A}$, then for $d=50 \cdot 10^{-3} \mathrm{~m}, I_{c r} \approx 600 \mathrm{~A}$, i.e. with increasing diameter $d$, the beginning of the critical current moves towards the high currents.

Analysis of photographs of the radial sections of the arc, obtained by a high-speed camera, shows a significant difference in behavior of the arc spots. Before $I_{\mathrm{cr}}$, one reference spot, uniformly moving in the electrode crosssection, can be observed. Then, after $I_{\mathrm{cr}}$, there are two spots simultaneously existing and moving with different velocities.
It was determined that the reasons for double arcing and sharp increase in specific erosion of the electrode are the loss of stability by the rotating gas flow and the cathode jet reaching the opposite electrode wall. It has been shown experimentally that a similar phenomenon is characteristic of the anode.

In Fig. 2, there is the experimental dependence of $\bar{G}$ on the velocity of the arc spot displacement $V_{\mathrm{s}}$ in regime $I<I_{\mathrm{cr}}$. The level of specific erosion of $10^{-9} \mathrm{~kg} / \mathrm{C}<\bar{G}<2 \cdot 10^{-9} \mathrm{~kg} / \mathrm{C}$, acceptable for practice, is achieved at relatively low velocities of anode arc spot displacement, which do not require the large gas flow rates.

The results of $V_{\varphi}$ measurements near the electrode wall (beyond the boundary layer) show that at $I<I_{\text {cr }}$ a slight decrease in $V_{\varphi}$ is observed with increasing current (Fig. 3). At $I<I_{\mathrm{cr}}$, the velocity of gas rotation drops sharply, and the flow in the electrode cavity loses its stability [4]. At that, $V_{\varphi}$ becomes uneven and indefinite, periodic cathode spot stops are observed, and average velocity of its displacement $V_{\mathrm{d}}$ decreases, which leads to growth in $\bar{G}$ (see Fig. 1).

$$
\bar{G}, \mathrm{~kg} / \mathrm{K}
$$

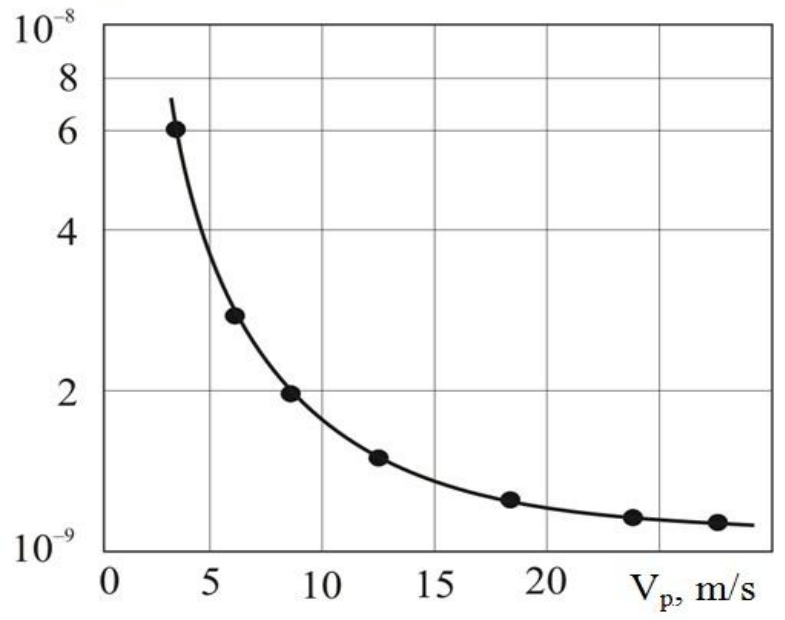

Fig. 2. Specific electrode erosion vs. velocity of cathode arc spot motion: $I=$ $420 \mathrm{~A}, G_{1}=G_{2}=5 \mathrm{~g} / \mathrm{s}, d=34 \mathrm{~mm}$

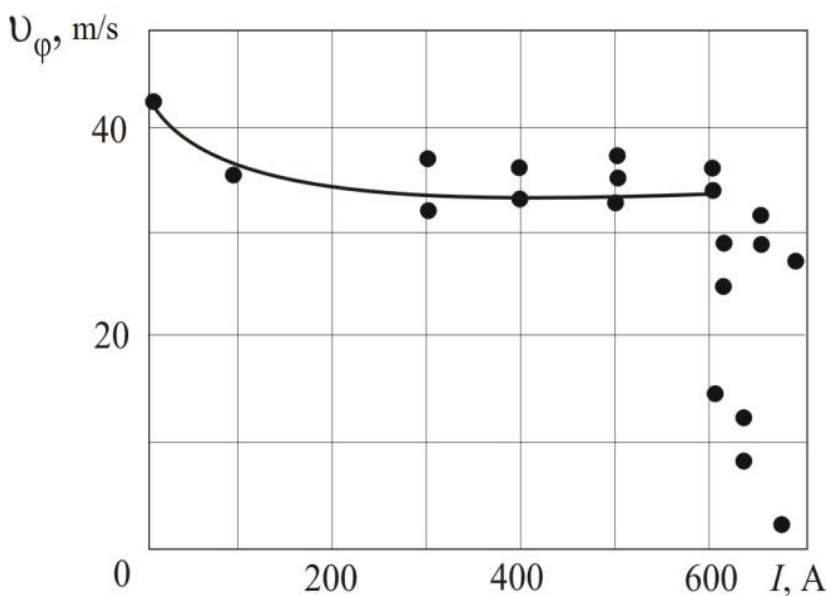

Fig. 3. Change in circumferencial component of gas velocity near electrode wall depending on arc current: $d=50 \mathrm{~mm} ; G_{1}=G_{2}=5 \mathrm{~g} / \mathrm{s}$ 
The stabilizing effect of the rotating flow of plasmaforming gas is determined by parameter $V_{\varphi}$. Since $V_{\varphi} \sim G / p d$, where $p$ is pressure, $\mathrm{Pa}$, values $G, p, d$ are easily measured and controlled, then ratio $G / p d$ should be considered one of the parameters influencing arc discharge stabilization.

III. AERODYNAMICS OF THE VORTEX FLOW IN THE ELECTRODE CHAMBER

Measurement results on axial $V_{z}$ and tangential $V_{\varphi}$ components of the flow velocity and static pressure $p$ over the electrode cross section (without electric current load) are shown in Fig. 4 for two electrode cross sections (inlet and outlet ones). According to the data presented, the gas flow in the electrode chamber is complex: both direct and reverse flows exist. Calculation of the mass gas flow rate by the values of $V_{z}$ and their comparison with measured values demonstrate that about $80 \%$ of gas introduced via the vortex chambers moves along the electrode wall. Gas with flow rote $G_{1}$ moves along coordinate $z$ towards the electrode outlet and gas with flow rate $G_{2}$ meets $G_{1}$. The place of their contact is called the zone of flow meeting, and at $I<I_{\mathrm{cr}}$, the radial region with the arc spot rotates over the electrode surface in this place (see Fig. 1, annular zone A).

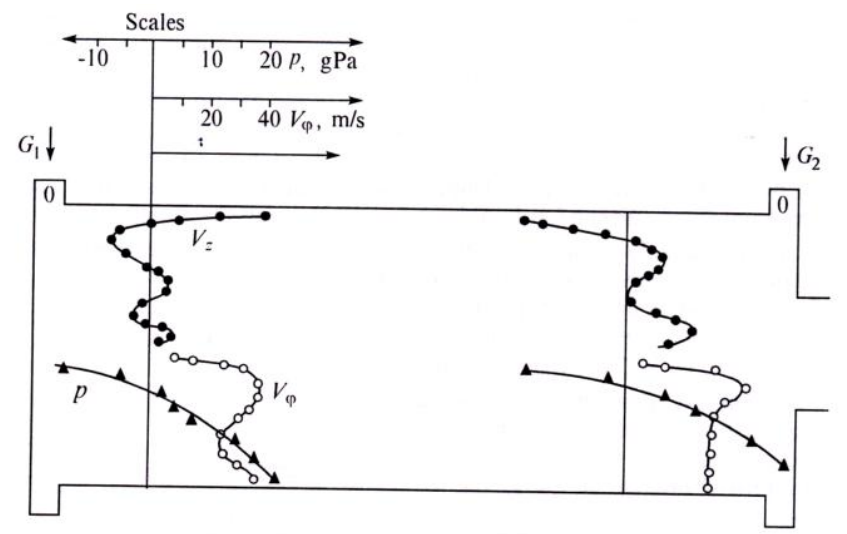

Fig. 4. Distribution of static pressure $p$, axial $V_{z}$ and tangential $V_{\varphi}$ velocity components inside the inner electrode.

$$
\mathrm{d}=7.26 \cdot 10^{-2} \mathrm{~m}, \mathrm{G}_{1}=\mathrm{G}_{2}=5 \cdot 10^{-3} \mathrm{~kg} / \mathrm{s} \text {. }
$$

The gas flow in the electrode chamber may be conventionally divided into two areas: the first is located between the back electrode lid and the place of flow $G_{1}$ and $G_{2}$ meeting place to the inter-electrode insertion. In both areas there are areas with axial gas circulation. In this connection, the main portion of gas before leaving the electrode travels long inside it. Since the turbulent viscosity of gas in the rotating flows is very high (approximately $100-1000$ times higher than the molecular one), this leads to a significant loss in velocity $V_{\varphi}$. It is obvious from Fig. 4 that despite the relatively high gas flow rate, the values of tangential velocity are low.

For the general case, the value of tangential velocity component in vortex chambers depends on many factors. The main of them are: gas flow rate $\mathrm{G}=\mathrm{G}_{1}+\mathrm{G}_{2}$ pressure $p$, electrode diameter $d$, ratio $D / d$ ( $\mathrm{D}$ is the diameter of the vortex ring), gas velocity at the outlet of vortex apparatus $V_{\varphi}$.
According to experience in plasmatorches with cylindrical electrodes, two last factors weakly affect the value of $V_{\varphi 0}$.

Analysis of experimental data on gas velocity measurements near the electrode wall showed that in the studied alteration range of parameters $G, p$ and $d$ :

$$
\mathrm{V}_{\varphi 0}=2.1 \cdot 10^{7} \mathrm{G} / \mathrm{pd} \text {. }
$$

When $2 \cdot 10^{-3} \leq \mathrm{G} \leq 24 \cdot 10^{-3} \mathrm{~kg} / \mathrm{s}, 10^{5} \leq \mathrm{p} \leq 3 \cdot 10^{5} \mathrm{~Pa}$, $2 \cdot 10^{-2} \leq \mathrm{d} \leq 7.2 \cdot 10^{-2} \mathrm{~m}, \mathrm{~V}_{\varphi} \geq 200 \mathrm{~m} / \mathrm{s}, 1.1 \leq \mathrm{D} / \mathrm{d} \leq 1.5$, formula accuracy makes up $\pm 10 \%$. This dependency is not universal and it is suitable only for vortex devices similar to the electrode known as a plasmatorch.

When studying parameters of the flame in a rotating flow, the stabilization criterion (Richardson number $\mathrm{Ri}$ ) was introduced in [6]. This criterion can also be used for the estimate of efficiency of arc stabilization in linear plasmatorches. There is a hot central gas flow stabilized by the rotating air flow inside the inner electrode. Measurements of temperature $T(r)$ near the radial arc region (closer to the end lid) demonstrated that the hot gas occupies not only the neararc area, but it also spreads towards the back part of the electrode. This is caused by axial gas circulation from the place of meeting gas flow $G_{1}$ and $G_{2}$ to the back lid of the electrode (see Fig. 4, curve $\mathrm{V}_{\mathrm{z}}$ ). Let us analyze the it from:

$$
R i=\frac{1}{p} \frac{\partial p}{\partial r} \frac{V_{\varphi}^{2}}{r} /\left[\left(\frac{\partial V_{\varphi}}{\partial r}\right)^{2}+\left(\frac{\partial V_{\varphi}}{\partial r}-W\right)^{2}\right],
$$

where $\left(\frac{\partial V_{\varphi}}{\partial r}-W\right)$ is the radial gradient of the tangential velocity component in the rotating coordinate system. Calculation of Ri number using experimental distribution of $V_{\varphi}(r), V_{z}(r)$ and $p(r)$ demonstrates that the vortex gas flow without arc (cold blowouts) is stable inside the whole electrode. Everywhere over cross section, $\mathrm{Ri}>0$; however, stabilizing effects are insignificant due to the low value of Ri.

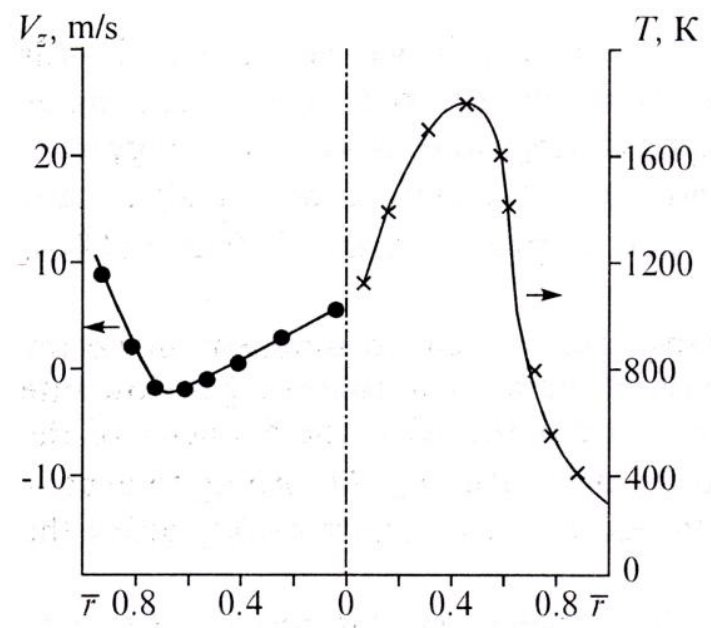

Fig. 5. Distribution of temperature $\mathrm{T}$ and axial component of velocity $\mathrm{V}_{\mathrm{z}}$ over electrode radius. 


$$
\mathrm{d}=5 \cdot 10^{-2} \mathrm{~m}, \mathrm{I}=500 \mathrm{~A} \text {. }
$$

In the presence of arc, in the near-axial area, $\mathrm{Ri}<0$. This is

caused by the fact that inside the electrode chamber at $\bar{r} \cong 0.7$ , there is the reverse flow from the arc to a back lid of the discharge chamber (Fig 5), and at the center of the electrode there is a cold flow from the back lid. Therefore, there is a depression at the center of temperature profile, and value $d p / d r$, which determines the sing of $\mathrm{Ri}$, becomes negative. Thus, the back central part of the electrode is the zone without stabilizing effect of the rotating flow. Transverse gas oscillation, which effects the stability of the whole hot flow in the electrode, may be developed there. However, in the rest part the $\mathrm{Ri}$, values are higher than at cold blowouts and the area with $\mathrm{Ri}>1$ exists. The stabilizing effect becomes predominant there.

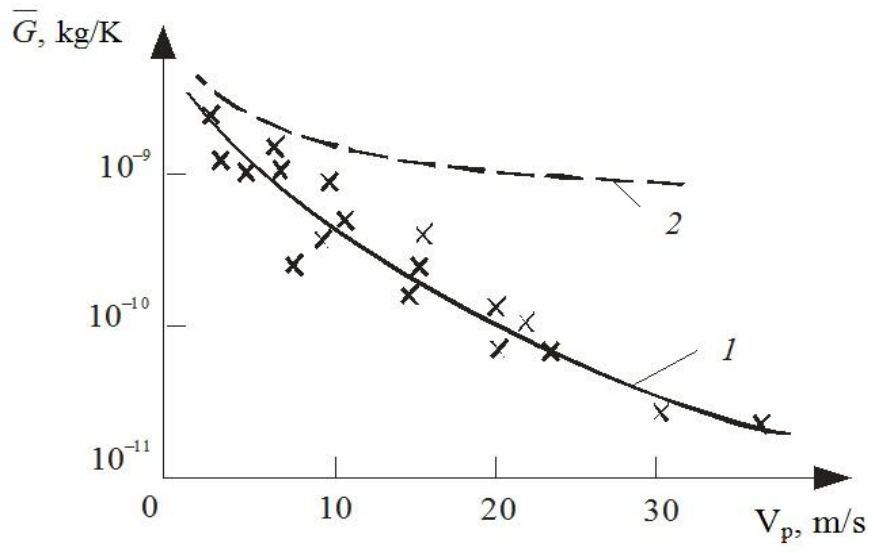

Fig. 6. Dependence of $\bar{G}$ of electrodes on arc spot velocity: $I=250 \mathrm{~A}, d=$ $34 \mathrm{~mm} ; 1$ - anode, 2 - cathode

Another parameter affecting the gas flow stability in the electrode cavity is the arc current. Since the length of the cathode jet increases with increasing current, starting from certain current $I=I_{\mathrm{cr}}$, it breaks through the stabilizing layer of the cold gas. Periodic breakdowns to the opposite part of the electrode occur. This causes powerful fluctuations of all flow parameters and contributes to the development of its instability. The higher $V_{\varphi}$, the greater the value of critical current $I_{\text {cr. }}$. Since $V_{\varphi} \sim G / p d$, then $I_{\text {cr }}=f(G, d, p)$ [7-9].

As for the cathode, specific erosion of anode depends on the velocity of the reference arc spot. Dependence of $\bar{G}$ on $V_{\mathrm{d}}$ for the anode in the air medium is presented in Fig. 4. At low values of $V_{\mathrm{d}}$, the level of specific erosion is about $10^{-9} \mathrm{~kg} / \mathrm{Cl}$ for both polarities of the electrodes. With increasing $V_{\mathrm{d}}$, the value of $\bar{G}$ for the anode decreases significantly, and at $V_{\mathrm{d}} \geq 30 \mathrm{~m} / \mathrm{s}$, it reaches the value of $2 \cdot 10^{-11} \mathrm{~kg} / \mathrm{C}$. Dependence $\bar{G}=f\left(V_{\mathrm{n}}\right)$ for the cathode is shown in Fig. 4 for comparison (see Fig. 2) [4]. These dependences can be explained by phenomenology of current transfer in the near-electrode regions of the cathode and anode. Thus, experimental data show that the loss of spatial stability by the arc discharge in the electrode cavity and the increase in its erosion are the interrelated phenomena, and at a given current, they are determined by aerodynamics of the gas flow and its stability.
Analysis of the equation of momentum conservation for the cathode jet [4] with the use of experimental data made it possible to determine the required conditions for the long service life of electrode in the air medium:

$$
G / p d \geq 2 \cdot 10^{-6} ; \quad I<I_{\mathrm{cr}}=1.6 \cdot 10^{6} \sqrt{G / p} .
$$

Relations (1) can be called the criteria for the working capacity of a copper tubular cathode: they allow us to estimate continuous operation of an electrode for calculated parameters $G, d, p$ even at the stage of plasmatron design.

Critical current $I_{\text {cr }}$ calculated by the second formula of (2) can be regarded as the limiting arc current, which should not be exceeded. Such current limitation is applied only to electrodes with diameter $d \leq 9 \cdot 10^{-2} \mathrm{~m}$.

Since the nature of the cathode and anode jets is the same, the working conditions of the copper cathode (2) can be similarly written for the anode:

$$
G / p d \geq 4 \cdot 10^{-6} ; \quad I<I_{\mathrm{cr}}=1.6 \cdot 10^{6}(G / p)^{1 / 2}
$$

The difference between first inequalities in (2) and (3) is caused by the fact that anode erosion depends more on arc spot velocity $V_{\mathrm{d}}$ than in the case of the cathode (Fig. 6). In addition, the rotation velocity of the anode spot under the same conditions is about 1.5 times lower than that of the cathode spot.

\section{ACKNOWLEDGMENTS}

The work was financially supported by the Ministry of Education and Science of the Russian Federation according to the Subsidiary Agreement No.14.607.21.0118 (unique project identifier RFMEFI60715X0118)

\section{LITERATURE REFERENCES}

[1] M.F. Zhukov, A.N. Timoshevskiy, A.N. Cherepanov, "Mechanisms of electrode erosion in linear dc plasmatorches" Thermophysics and Aeromechanics, Vol.4, No. 2, pp. 227-237, 1997.

[2] V.I. Golish, E.I. Karpenko, V.G. Luk'yashchenko, V.E. Messerle, A.B. Ustimenko, V.Z. Ushanov "Long-service-life plasma arc torch" High Energy Chemistry, Vol.43 No.4, pp. 318-323, 2009.

[3] A.S. Anshakov, A.N. Timoshevskiy, E.K. Urbakh. Izv. SO AN SSSR, 2 1988.

[4] A.S. Anshakov, A.N. Bykov, A.N. Timoshevskiy, E.K. Urbakh "Interrelation of erosion processes with aerodynamics of a swirling flow in a cylindrical electrode of a plasma torch"Thermophysics and Aeromechanics, No.4, pp.597, 2002.

[5] J.N. Beer, N.A. Chigier et all Combus, Flame 1, 1971.

[6] V.S. Cherednichenko, A.S. Anshakov, M.G. Kuzmin. Plasma ElectricProcess Installations: Textbook for High Schools. NSTU, Novosibirsk, 2011 .

[7] E.K. Urbahk, "Development and Study of Technologikal Electric-arc Plasmatorch", abstr. Of Doctoral Thesis, Novosibirsk, 1999.

[8] A.S.Anshakov, P.V. Domarov, V.A. Faleev, A.A. Danilenko, "Modeling ohmic heating in the drying zone of the plasma shaft electric furnace, when recycling the technogenic waste", JOP: CS, Vol.830, No.1, 2017

[9] A.S. Anshakov, P.V. Domarov, S.I. Radko, V.A. Faleev, "Development of arc plasmatrons with long service life of electrodes", IFOST 2016 7884271, pp.37-40, 2017. 
[10] A.S. Anshakov, V.A. Faleev, M.V. Cherednichenko, "Methods for increasing the life of electrodes in arc plasmatrons" $\mathrm{T}$ and A, Vol.24, No.1, pp.141, 2017.

[11] A.I. Aliferov, A.S. Anshakov, V.A. Sinitsyn, "Numerical modelling of heat transfer in plasma shaft electric furnace at utilization of anthropogenic waste" Thermophysics and Aeromechanics, Vol.16, No.1, pp. 155-161,2009.
[12] I.B.Georgiev, B.I. Mikhailov, "Coal gasification in the water-steam plasma" High Energy Chem., Vol. 25, No.4, pp. 76-80, 1991.

[13] B.I. Mikhailov, "Electric arch generators of watersteam plasma". Part 2 Thermophysics and Aeromechanics, Vol.10, No.4, pp. 637-657, 2003.

[14] Hyun-Seo Park. "Study of slag content and properties after plasma melting of incineration ash" Thermophysics and Aeromechanics, Vol 18, No. 2, pp. 313-321, 2011. 